\title{
Vias de formação, estabilidade e características químicas de agregados em solos sob sistemas de manejo agroecológico
}

\author{
Celeste Queiroz Rossi ${ }^{(1)}$, Marcos Gervasio Pereira(1), Octavio Vioratti Telles de Moura ${ }^{(1)}$ \\ e Anastacia Perci Campos de Almeida ${ }^{(1)}$
}

\begin{abstract}
(1)Universidade Federal Rural do Rio de Janeiro, Departamento de Solos, BR-465, Km 7, CEP 23890-000 Seropédica, RJ, Brasil. E-mail: celesteqrossi@yahoo.com.br, gervasio@ufrrj.br, octavio.vioratti@gmail.com, anastaciaperci@gmail.com
\end{abstract}

Resumo - O objetivo deste trabalho foi identificar as vias de formação de agregados biogênicos, intermediários e fisiogênicos, bem como sua estabilidade e seus atributos químicos em diferentes sistemas de manejo agroecológico. Foram escolhidas cinco áreas para coleta de amostras, em Argissolo Vermelho-Amarelo, no Município de Seropédica, RJ: SAF, sistema agroflorestal, com 10 anos de implantação; C-SOL, café a pleno sol, com 15 anos; C-SOM, café sombreado, com 15 anos; AL-FLE, aleias com flemíngia (Flemingia macrophylla), com 10 anos; e PD, sistema plantio direto, com milho e berinjela, com 6 anos. As amostras foram coletadas às profundidades de $0,00-0,05$ e $0,05-0,10 \mathrm{~m}$, e a fração dessas amostras retida em peneiras de 9,7 e $8,0 \mathrm{~mm}$ foi utilizada para identificar os diferentes tipos de agregado. Após a separação, foram avaliados a estabilidade e os atributos químicos de cada tipo. Os sistemas de manejo não produziram efeito sobre a proporção média de cada tipo de agregado, e a contribuição relativa dos agregados intermediários para a massa total de agregados foi superior à dos demais. Os sistemas C-SOL e C-SOM apresentam os maiores teores de carbono orgânico total, carbono orgânico associado aos minerais, carbono orgânico particulado, independentemente dos sistemas e das profundidades avaliadas.

Termos para indexação: agregados fisiogênicos, carbono orgânico total, fracionamento da matéria orgânica, qualidade do solo, sistemas agroflorestais, vias de formação de agregados.

\section{Formation pathways, stability and chemical characteristics of soil aggregates in agroecological management systems}

\begin{abstract}
The objective of this work was to identify the formation pathways of biogenic, intermediate, and physiogenic aggregates, as well as their stability and chemical characteristics in different agroecological management systems. Five areas were chosen for sampling in Argissolo Vermelho-Amarelo (Ultisol), in the municipality of Seropédica, in the state of Rio de Janeiro, Brazil: SAF, 10-year agroforestry system; C-SOL, 15-year full-sun coffee; C-SOM, 15-year shaded coffee; AL-FLE, 10-year alleys with flemingia (Flemingia macrophylla); and PD, 6-year no-tillage system with corn and eggplant. Soil samples were collected at the $0.00-0.05$ and $0.05-0.10-\mathrm{m}$ soil depths, and the fraction retained in sieves of 9.7 and $8.0 \mathrm{~mm}$ were used to identify the different types of aggregates. After separation, the stability and chemical characteristics of each aggregate type were evaluated. The proportion between the aggregate types was not affected by the managements systems, and the relative contribution of intermediate aggregates to the total aggregate mass was higher than that of the other ones. The C-SOL and C-SOM systems show the highest total organic carbon, organic carbon associated with minerals, and particulate organic carbon, irrespectively of the evaluated systems and depths.
\end{abstract}

Index terms: physiogenic aggregates, total organic carbon, organic matter fractioning, soil quality, agroforestry systems, aggregate formation pathways.

\section{Introdução}

A utilização de práticas conservacionistas de manejo do solo tem recebido grande ênfase ultimamente, basicamente no que se refere à manutenção e à melhoria dos atributos físicos, químicos e biológicos dos solos cultivados, e suas implicações quanto ao rendimento das culturas (Argenton et al., 2005). Nesse contexto, os atributos edáficos podem ser utilizados para avaliar a relação entre o manejo e a qualidade do solo (Lima et al., 2007).

A estrutura é um dos principais atributos do solo, importante principalmente no que tange à dinâmica 
da água e ao crescimento vegetal. Ela está relacionada à organização das partículas minerais no solo, que podem estar individualizadas ou em agregados, formados a partir dos processos de aproximação e cimentação dessas partículas, ambos destacadamente influenciados pela matéria orgânica do solo (MOS) (Six et al., 2004; Mulumba \& Lal, 2008; Noellemeyer et al., 2008; Brady \& Weil, 2013). De acordo com o manejo agrícola adotado, a MOS pode receber maior proteção física contra a rápida decomposição, em agregados mais estáveis (Pulleman \& Marinissen, 2004; Fernandéz et al., 2010).

A estreita ligação entre a fauna do solo, a decomposição e estabilização da matéria orgânica (MOS) e a agregação do solo já foi reconhecida e é intensamente estudada (Six et al., 2004; Rilling \& Mummey, 2006). Essa agregação pode ser influenciada por diversos fatores: organismos do solo; raízes; agentes inorgânicos, manejo adotado e também por variáveis ambientais. As interações entre esses fatores resultam na formação e estabilização dos agregados (Six et al., 2004; Bronick \& Lal, 2005; Rilling \& Mummey, 2006).

Os agregados podem ser classificados morfologicamente em biogênicos, fisiogênicos, ou intermediários a estes dois tipos (Batista, 2011). A influência de fatores químicos, a adição de matéria orgânica ao solo e os ciclos de umedecimento e secagem constituem uma via lenta de formação dos agregados denominados fisiogênicos. No entanto, quando ocorre a participação significativa da fauna do solo, dos microrganismos e também das raízes das plantas, a via de formação dos agregados é denominada de biogênica. Quando não se observa uma clara distinção entre essas duas vias, os agregados são considerados intermediários (Batista, 2011).

Estudos dos diferentes tipos de agregados (biogênicos, intermediários e fisiogênicos), em diferentes sistemas de produção agroecológica, são relevantes para ampliar o conhecimento da dinâmica de formação desses agregados e, principalmente, para avaliação de seu papel como potenciais indicadores da qualidade do solo (Silva Neto et al., 2010).

O objetivo deste trabalho foi identificar as vias de formação de agregados biogênicos, intermediários e fisiogênicos, bem como sua estabilidade e seus atributos químicos em diferentes sistemas de manejo agroecológico.

\section{Material e Métodos}

O estudo foi realizado na área do Sistema Integrado de Produção Agroecológica, Sipa, denominado Fazendinha Agroecológica do Km 47 (Almeida et al., 1999). A área está localizada na Embrapa Agrobiologia, em Seropédica, RJ, a $22^{\circ} 45^{\prime} \mathrm{S} 43^{\circ} 41^{\prime} \mathrm{W}$, e à altitude de $33 \mathrm{~m}$, com clima do tipo Aw (Neves et al., 2005). O solo foi classificado como Argissolo Vermelho-Amarelo (Santos et al., 2013), está localizado em área com relevo suave-ondulado e vem sendo rotineiramente cultivado com oleráceas e frutíferas.

Foram selecionadas cinco áreas sob manejo agroecológico, com o seguinte histórico de uso: SAF, sistema agroflorestal, com 10 anos de implantação, formado por banana (Musa sapientum), palmito-jussara (Euterpe oleracea), cacau (Thebroma cacao), mamão (Carica papaya), guapuruvu (Schizolobium parahyba), urucum (Bixa orellana L) e açaí (Euterpe olearacea); C-SOL, cultivo perene, com 15 anos de café (Coffea canephora) a pleno sol; C-SOM, cultivo perene, com 15 anos de café ( $C$. canephora) sombreado com gliricídia (Gliricidia sepium); AL-FLE, cultivo em aleias, com 10 anos de flemingia (Flemingia macrophylla) e com vagem (Phaseolus vulgaris var. vulgaris); e PD, plantio direto, com 6 anos de milho (Zea mays)/berinjela (Solanum melongena). As áreas de café recebem, anualmente, adubação na cova com "bokashi", e a área com PD tem recebido adubação em sulco, no momento do plantio, com esterco de curral (doses necessárias para aportes de 50 a $100 \mathrm{~kg} \mathrm{ha}^{-1} \mathrm{de}$ $\mathrm{N}$ ), e cama de aviário, em cobertura (doses entre 100 a $200 \mathrm{~kg} \mathrm{ha}^{-1}$ de N).

A coleta de amostras para o estudo da gênese dos agregados foi realizada em maio de 2014 . Foram retiradas amostras das camadas de $0,00-0,05$ e $0,05-0,10 \mathrm{~m}$, mediante a abertura de trincheiras transversais às linhas de semeadura, tendo-se avaliado a fração das amostras retida no intervalo de peneiras entre 9,7 e $8,0 \mathrm{~mm}$.

A contribuição relativa, em massa, de cada tipo de agregado foi determinada com a pesagem de $100 \mathrm{~g}$ de agregados de cada parcela experimental. Os agregados foram observados sob lupa binocular e separados à mão, de acordo com as definições de Bullock et al. (1985). A separação dos agregados foi feita por meio de padrões morfológicos, da seguinte forma: agregados fisiogênicos, identificados por apresentarem formas angulares; biogênicos, apresentam formas arredondadas resultantes da passagem pelo trato intestinal dos 
indivíduos da macrofauna do solo, principalmente Oligochaeta (minhocas), ou identificados pela visualização de raízes; e intermediários, caracterizados por possuírem formas indefinidas. Esses agregados podem ter passado pela via biogênica de formação, mas tiveram perda da forma arredondada pelo envelhecimento ou pela via fisiogênica.

Após a separação, os agregados foram submetidos à análise de estabilidade em água, tendo-se utilizado a técnica de tamisação vertical, em conjunto de peneiras com malha decrescente, a saber: 2,00, 1,00, 0,50, 0,25 e $0,105 \mathrm{~mm}$, por $15 \mathrm{~min}$, no aparelho de Yooder. Passado o tempo, o material retido em cada peneira foi colocado em placas de Petri previamente pesadas, e levado à estufa, a $65^{\circ} \mathrm{C}$, até obtenção de massa constante. A partir dos dados de massa de agregados, foi calculado seu diâmetro médio ponderado (DMP) e diâmetro médio geométrico (DMG), conforme Claessen (1997). Para a realização das demais análises, os agregados foram destorroados e passados em peneira de $2 \mathrm{~mm}$, para a obtenção da terra fina seca ao ar (TFSA). A determinação do carbono orgânico total seguiu a metodologia de Yeomans \& Bremner (1988), e a dos teores de $\mathrm{Ca}^{2+}, \mathrm{Mg}^{2+}, \mathrm{K}^{+}, \mathrm{Na}^{+}, \mathrm{Al}^{3+}, \mathrm{H}+\mathrm{Al}, \mathrm{P}$ e $\mathrm{pH}$ em água foi feita conforme Claessen (1997). O fracionamento granulométrico foi realizado segundo Cambardella \& Elliott (1992).

Os resultados foram analisados quanto à normalidade e homogeneidade, por meio dos testes de Lilliefors, e Cochran e Barttlet, respectivamente. $\mathrm{O}$ experimento foi analisado conforme delineamento inteiramente casualizado, com cinco tratamentos, duas profundidades e quatro repetições. Os resultados foram submetidos à análise de variância, e os valores médios, quando significativos, foram comparados entre si pelo teste Scott-Knott, a 5\% de probabilidade. Também foi realizada a análise de componentes principais (ACP), para uma melhor interpretação do padrão dos agregados em função dos atributos avaliados, por meio do programa XL Stat, e análise de correlação de Pearson pelo programa Excel.

\section{Resultados e Discussão}

Os sistemas de manejo agroecológico não produziram efeito significativo sobre as proporções relativas dos diferentes tipos de agregados (Figura 1). Houve predominância de agregados intermediários, independentemente das profundidades e dos sistemas de manejo avaliados. Silva Neto et al. (2012) também não observaram diferenças quanto à composição relativa de agregados biogênicos, intermediários e fisiogênicos, entre áreas com diferente cobertura vegetal, mas relataram maior contribuição de agregados fisiogênicos na massa total dos agregados.

Batista et al. (2013) constataram que as épocas do ano têm efeito significativo sobre a proporção relativa de agregados, com predominância de agregados intermediários na época seca, e predominância variável conforme o manejo, na época chuvosa. Em estudo com
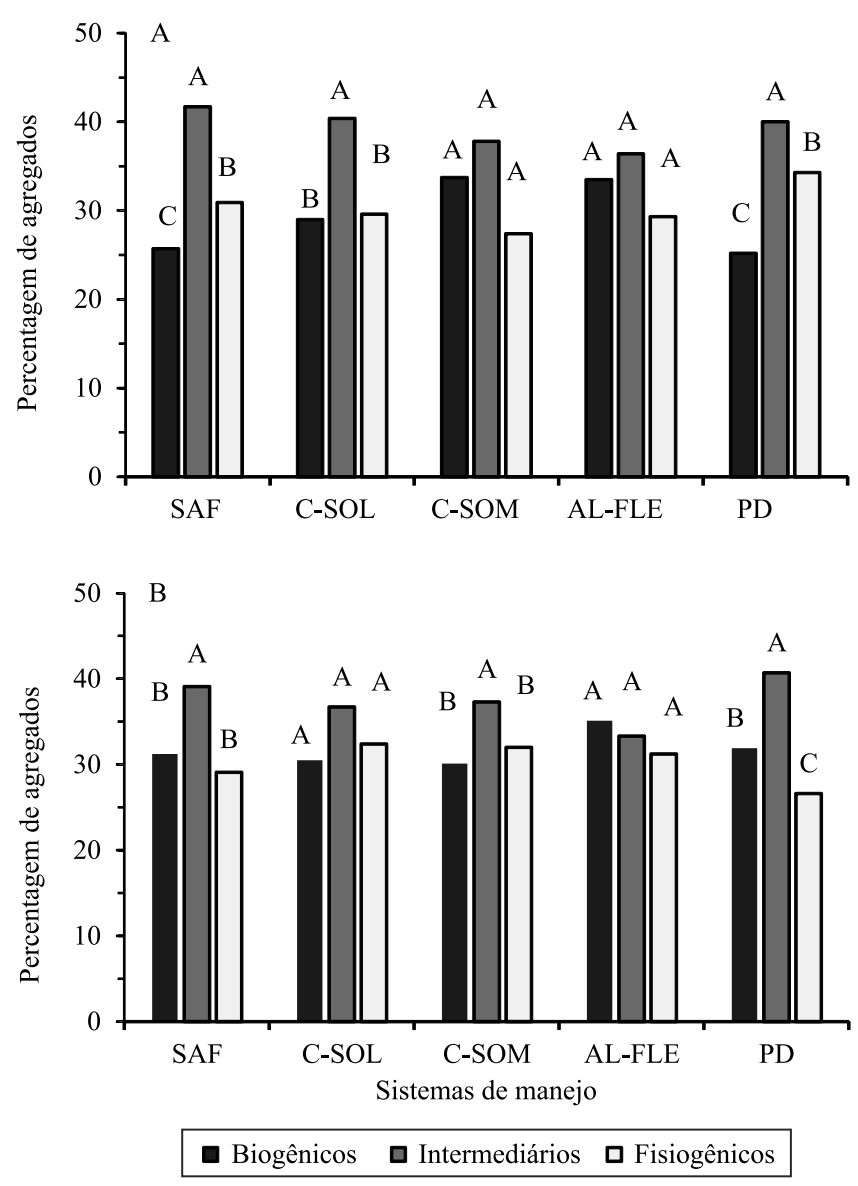

Figura 1. Percentagem média de agregados biogênicos, intermediários e fisiogênicos, em sistemas de manejo agroecológico, às camadas de 0,00-0,05 (A) e 0,05-0,10 m (B). Médias seguidas de letras iguais, na comparação entre tipos de agregado, nos diferentes sistemas de manejo, não diferem pelo teste de Scott-Knott, a 5\% de probabilidade. SAF, sistema agroflorestal; C-SOL, café cultivado a pleno sol; C-SOM, café sombreado; AL-FLE, cultivo em aleias; $\mathrm{PD}$, sistema plantio direto. 
fertilidade e frações da matéria orgânica de agregados, Loss et al. (2014) verificaram que, independentemente do manejo, agregados fisiogênicos sempre apresentam maiores proporções relativas do que os biogênicos, até $0,10 \mathrm{~m}$ de profundidade; no entanto, em cultivo convencional do solo, os autores somente relataram presença de agregados fisiogênicos. Os resultados encontrados por Pulleman etal.(2005) tambémmostram a forte influência do plantio convencional na formação de agregados fisiogêncios. Os autores verificaram que $67 \%$ da massa de agregados, na camada de $0,10 \mathrm{~m}$, é contituída por agregados fisiogênicos, e que apenas $7,4 \%$ é constituída por agregados biogênicos, em área com longo histórioco de plantio convencional. Portanto, espera-se que sistemas conservacionistas aumentem a estabilização do carbono pela maior formação e manutenção de agregados biogênicos (Brussaard et al., 2007).

Os resultados de DMP e DMG sofreram influência significativa dos manejos somente quanto aos agregados fisiogênicos e intermediários (Tabela 1). Na área com PD, verificaram-se os menores valores de DMP e DMG, para ambos os tipos de agregados. O cultivo de oleráceas, mesmo em sistema de $\mathrm{PD}$, apresenta uso mais intenso de práticas culturais do que os demais

Tabela 1. Diâmetro médio ponderado (DMP) e de diâmetro médio geométrico (DMG), em agregados biogênicos (BIO), intermediários (INT) e fisiogênicos (FIS), nos sistemas de manejo agroecológico ${ }^{(1)}$.

\begin{tabular}{|c|c|c|c|c|c|c|}
\hline \multirow[t]{2}{*}{ Sistema } & \multicolumn{3}{|c|}{$\mathrm{DMP}(\mathrm{mm})$} & \multicolumn{3}{|c|}{$\mathrm{DMG}(\mathrm{mm})$} \\
\hline & $\mathrm{BIO}$ & INT & FIS & $\mathrm{BIO}$ & INT & FIS \\
\hline & \multicolumn{6}{|c|}{$0,00-0,05 \mathrm{~m}$} \\
\hline SAF & $4,5^{\mathrm{ns}}$ & $4,2 \mathrm{~A}$ & $4,4 \mathrm{~A}$ & $3,7^{\mathrm{ns}}$ & $3,1 \mathrm{~B}$ & $3,4 \mathrm{~A}$ \\
\hline C-SOL & 4,6 & $4,6 \mathrm{~A}$ & $4,7 \mathrm{~A}$ & 3,7 & $3,9 \mathrm{~A}$ & $3,9 \mathrm{~A}$ \\
\hline C-SOM & 4,1 & $4,1 \mathrm{~A}$ & $4,0 \mathrm{~A}$ & 3,0 & $3,0 \mathrm{~B}$ & $3,0 \mathrm{~B}$ \\
\hline AL-FLE & 4,4 & $4,2 \mathrm{~A}$ & $4,6 \mathrm{~A}$ & 3,6 & $3,8 \mathrm{~A}$ & $4,1 \mathrm{~A}$ \\
\hline PD & 4,5 & $2,9 \mathrm{~B}$ & $3,0 \mathrm{~B}$ & 3,8 & $1,6 \mathrm{C}$ & $2,2 \mathrm{~B}$ \\
\hline \multirow[t]{2}{*}{ CV (\%) } & 6,2 & 9,3 & 9,4 & 12,5 & 15,2 & 12,3 \\
\hline & \multicolumn{6}{|c|}{$0,05-0,10 \mathrm{~m}$} \\
\hline SAF & $4,2^{\mathrm{ns}}$ & $4,0 \mathrm{~A}$ & $3,9 \mathrm{~A}$ & $3,3 \mathrm{~B}$ & $2,9 \mathrm{~A}$ & $2,9 \mathrm{~A}$ \\
\hline C-SOL & 4,7 & $4,3 \mathrm{~A}$ & $4,3 \mathrm{~A}$ & $4,3 \mathrm{~A}$ & $3,4 \mathrm{~A}$ & $3,1 \mathrm{~A}$ \\
\hline C-SOM & 4,2 & $3,9 \mathrm{~A}$ & $4,3 \mathrm{~A}$ & $3,2 \mathrm{~B}$ & $3,1 \mathrm{~A}$ & $3,4 \mathrm{~A}$ \\
\hline AL-FLE & 4,9 & $3,4 \mathrm{~B}$ & $3,6 \mathrm{~A}$ & $2,6 \mathrm{~B}$ & $2,0 \mathrm{~B}$ & $2,4 \mathrm{~B}$ \\
\hline PD & 3,3 & $2,1 \mathrm{C}$ & $3,1 \mathrm{~A}$ & $2,5 \mathrm{~B}$ & $1,1 \mathrm{C}$ & $2,6 \mathrm{~B}$ \\
\hline CV (\%) & 26 & 9,8 & 14,9 & 20,5 & 22,6 & 14,4 \\
\hline
\end{tabular}

${ }^{(1)}$ Médias seguidas de letras iguais, nas colunas, não diferem pelo teste Scott-Knott, a $5 \%$ de probabilidade. ns Não significativo a $5 \%$ de probabilidade. SAF, sistema agroflorestal; C-SOL, café cultivado a pleno sol; C-SOM, café sombreado; AL-FLE, cultivo em aleias; PD, sistema plantio direto. sistemas de manejo agroecológico avaliados, o que favorece a decomposição de matéria orgânica, uma menor estabilidade de agregados e, consequentemente, a redução nos valores de DMP e DMG.

Silva Neto et al. (2012) relataram menores valores de DMP e DMG em área de pasto misto, em comparação à área de floresta secundária em estádio avançado de regeneração. Batista et al. (2013), entretanto, não observaram diferenças estatísticas para DMP e DMG, na estação seca, entre diferentes cultivos no Cerrado. $\mathrm{Na}$ estação chuvosa, os autores constataram menor média para a rotação algodão/soja, em comparação a outros cultivos.

Em geral, os diferentes manejos apresentaram boa reserva de nutrientes e ausência de elementos tóxicos em seus agregados (Tabela 2). Os valores de $\mathrm{pH}$ em água foram superiores a 5,4, com destaque para área de $\mathrm{PD}$, que apresentou os maiores valores de $\mathrm{pH}$ e menores de $\mathrm{H}+\mathrm{Al}$, independentemente da profundidade. Os sistemas C-SOL, C-SOM e SAF apresentaram as maiores disponibilidades de $\mathrm{Ca}^{2+}+$ $\mathrm{Mg}^{2+}$, e de $\mathrm{Ca}^{2+}$, nos três tipos de agregados, seguidos pelos sistemas AL-FLE e PD. Esses resultados evidenciam a importância de sistemas perenes para o aporte e a manutenção de material orgânico no solo, com reflexos no suprimento de nutrientes pelo solo. Nas áreas com culturas perenes, observaram-se maiores quantidades de coprólitos, que apresentam elevados teores de cálcio em sua composição (Schrader \& Zhang, 1997). Estudos como os de Silva Neto et al. (2010) e Loss et al. (2014) indicam que áreas com mais agregados biogênicos (coprólitos) apresentam maiores teores de $\mathrm{Ca}^{2+}$ do que áreas com maiores proporções de agregados fisiogênicos. As glândulas calcíferas encontradas em minhocas contribuem para o enriquecimento do material no solo e levam à formação de agregados com maiores teores do elemento (Schrader \& Zhang, 1997).

Os teores de $\mathrm{P}$ não apresentaram diferenças entre os sistemas estudados, à camada de $0,00-0,05 \mathrm{~m}$, enquanto os de $\mathrm{K}$ foram maiores no sistema $\mathrm{C}-\mathrm{SOL}$, à profundidade de $0,00-0,05 \mathrm{~m}$, e no $\mathrm{SAF}$, à profundidade de $0,05-0,10 \mathrm{~m}$. Nessa camada mais profunda, os valores observados para o nutriente foram considerados muito altos também nos sistemas C-SOL e C-SOM. Vale ressaltar que o Ké requerido em grandes quantidades pela cultura do café, principalmente na formação dos grãos (Matiello et al., 2006). 
Os valores de carbono orgânico total (COT), apresentados na Tabela 3, não diferiram significativamente entre os tipos de agregados, em nenhuma das áreas estudadas. Esse resultado pode se justificar pelo fato de que todos os sistemas avaliados são conservacionistas, e promovem o acúmulo e a

Tabela 2. Atributos químicos dos agregados biogênicos, intermediários e fisiogênicos, nos diferentes sistemas de manejo agroecológico avaliados e nas diferentes camadas de profundidades ${ }^{(1)}$.

\begin{tabular}{|c|c|c|c|c|c|c|c|c|c|c|}
\hline \multirow[t]{2}{*}{ Sistema } & \multirow{2}{*}{$\begin{array}{c}\mathrm{pH} \\
\mathrm{H}_{2} \mathrm{O} \\
\end{array}$} & $\mathrm{H}+\mathrm{Al}$ & $\mathrm{Ca}+\mathrm{Mg}$ & $\mathrm{Ca}$ & $\mathrm{Na}$ & $\mathrm{S}$ & $\mathrm{T}$ & \multirow{2}{*}{$\begin{array}{c}\mathrm{V} \\
(\%)\end{array}$} & \multirow{2}{*}{\multicolumn{2}{|c|}{$\begin{array}{c}\mathrm{P} \\
-\end{array}$}} \\
\hline & & \multicolumn{6}{|c|}{ - } & & & \\
\hline & \multicolumn{10}{|c|}{ Biogênico, camada de $0,00-0,05 \mathrm{~m}$} \\
\hline SAF & $5,9 \mathrm{~A}$ & $2,4 \mathrm{~A}$ & $5,0 \mathrm{~A}$ & $3,0 \mathrm{~A}$ & $0,0^{\mathrm{ns}}$ & $5,3 \mathrm{~B}$ & $7,7 \mathrm{~A}$ & $69,4 \mathrm{~B}$ & $42,5^{\mathrm{ns}}$ & $125,8 \mathrm{~B}$ \\
\hline C-SOL & $6,0 \mathrm{~A}$ & $2,1 \mathrm{~A}$ & $6,7 \mathrm{~A}$ & $3,1 \mathrm{~A}$ & 0,0 & $7,4 \mathrm{~A}$ & $9,5 \mathrm{~A}$ & $78,4 \mathrm{~B}$ & 35,4 & $285,7 \mathrm{~A}$ \\
\hline C-SOM & $6,0 \mathrm{~A}$ & $1,7 \mathrm{~A}$ & $5,5 \mathrm{~A}$ & $2,8 \mathrm{~A}$ & 0,0 & $5,6 \mathrm{~B}$ & $7,3 \mathrm{~A}$ & $77,0 \mathrm{~B}$ & 25,0 & $64,4 \mathrm{~B}$ \\
\hline AL-FLE & $5,3 \mathrm{~A}$ & $1,5 \mathrm{~A}$ & $2,8 \mathrm{~B}$ & $1,6 \mathrm{~B}$ & 0,0 & $2,9 \mathrm{C}$ & $4,4 \mathrm{~B}$ & $65,3 \mathrm{~B}$ & 35,5 & $44,9 \mathrm{~B}$ \\
\hline PD & $6,4 \mathrm{~A}$ & $0,5 \mathrm{~B}$ & $2,6 \mathrm{~B}$ & $1,6 \mathrm{~B}$ & 0,0 & $2,8 \mathrm{C}$ & $3,2 \mathrm{~B}$ & $86,1 \mathrm{~A}$ & 44,7 & $56,6 \mathrm{~B}$ \\
\hline \multirow[t]{2}{*}{ CV (\%) } & 5,8 & 23,5 & 25,4 & 26,9 & 19,6 & 24,7 & 21,7 & 12,0 & 19,5 & 26,5 \\
\hline & \multicolumn{10}{|c|}{ Intermediário, camada de $0,00-0,05 \mathrm{~m}$} \\
\hline SAF & $5,9 \mathrm{~B}$ & $2,2 \mathrm{~A}$ & $4,5 \mathrm{~A}$ & $3,0 \mathrm{~A}$ & $0,0^{\text {ns }}$ & $4,9 \mathrm{~B}$ & $7,1 \mathrm{~A}$ & $68,8 \mathrm{~B}$ & $29,5^{\text {ns }}$ & $117,0 \mathrm{~B}$ \\
\hline $\mathrm{C}-\mathrm{SOL}$ & $6,1 \mathrm{~B}$ & $2,0 \mathrm{~A}$ & $6,2 \mathrm{~A}$ & $3,3 \mathrm{~A}$ & 0,0 & $6,9 \mathrm{~A}$ & $8,9 \mathrm{~A}$ & $77,4 \mathrm{~A}$ & 35,5 & $267,2 \mathrm{~A}$ \\
\hline C-SOM & $6,0 \mathrm{~B}$ & $1,6 \mathrm{~A}$ & $5,2 \mathrm{~A}$ & $2,5 \mathrm{~A}$ & 0,0 & $5,4 \mathrm{~B}$ & $7,0 \mathrm{~A}$ & $77,3 \mathrm{~A}$ & 27,7 & $58,5 \mathrm{~A}$ \\
\hline AL-FLE & $5,3 \mathrm{~B}$ & $1,5 \mathrm{~A}$ & $3,1 \mathrm{~B}$ & $1,5 \mathrm{~B}$ & 0,0 & $3,1 \mathrm{C}$ & $4,6 \mathrm{~B}$ & $67,7 \mathrm{~B}$ & 39,4 & $37,1 \mathrm{~A}$ \\
\hline PD & $6,4 \mathrm{~A}$ & $0,5 \mathrm{~B}$ & $2,6 \mathrm{~B}$ & $1,6 \mathrm{~B}$ & 0,0 & $2,8 \mathrm{C}$ & $3,2 \mathrm{~B}$ & $86,1 \mathrm{~A}$ & 50,6 & $58,5 \mathrm{~A}$ \\
\hline \multirow[t]{2}{*}{ CV (\%) } & 3,8 & 17,8 & 22,00 & 15,7 & 20,3 & 23,3 & 21,1 & 9,1 & 19,7 & 24,3 \\
\hline & \multicolumn{10}{|c|}{ Fisiogênico, camada de $0,00-0,05 \mathrm{~m}$} \\
\hline SAF & $5,9 \mathrm{C}$ & $2,4 \mathrm{~A}$ & $4,4 \mathrm{~A}$ & $2,6 \mathrm{~B}$ & $0,0^{\mathrm{ns}}$ & $4,7 \mathrm{~B}$ & $7,0 \mathrm{~A}$ & $66,3 \mathrm{~B}$ & $36,5^{\mathrm{ns}}$ & $106,3 \mathrm{~B}$ \\
\hline C-SOL & $6,2 \mathrm{~B}$ & $1,8 \mathrm{~A}$ & $5,8 \mathrm{~A}$ & $3,5 \mathrm{~A}$ & 0,0 & $6,4 \mathrm{~A}$ & $8,1 \mathrm{~A}$ & $78,5 \mathrm{~A}$ & 46,9 & $234,0 \mathrm{~A}$ \\
\hline C-SOM & $6,0 \mathrm{~B}$ & $1,3 \mathrm{~A}$ & $4,7 \mathrm{~A}$ & $2,5 \mathrm{~B}$ & 0,0 & $4,8 \mathrm{~B}$ & $6,1 \mathrm{~A}$ & $79,1 \mathrm{~A}$ & 27,3 & $54,6 \mathrm{~B}$ \\
\hline AL-FLE & $5,3 \mathrm{C}$ & $1,5 \mathrm{~A}$ & $2,4 \mathrm{~B}$ & $1,5 \mathrm{C}$ & 0,0 & $2,5 \mathrm{C}$ & $4,0 \mathrm{~B}$ & $63,4 \mathrm{~B}$ & 36,8 & $33,2 \mathrm{~B}$ \\
\hline PD & $6,4 \mathrm{~A}$ & $0,4 \mathrm{~B}$ & $2,6 \mathrm{~B}$ & $1,5 \mathrm{C}$ & 0,0 & $2,7 \mathrm{C}$ & $3,2 \mathrm{~B}$ & $86,6 \mathrm{~A}$ & 39,3 & $54,6 \mathrm{~B}$ \\
\hline \multirow[t]{2}{*}{ CV (\%) } & 3,3 & 19,7 & 23,6 & 20,9 & 23,5 & 22,3 & 20,3 & 9,8 & 17,5 & 26,5 \\
\hline & \multicolumn{10}{|c|}{ Biogênico, camada de $0,05-0,10 \mathrm{~m}$} \\
\hline SAF & $6,0 \mathrm{~B}$ & $2,4 \mathrm{~A}$ & $4,0 \mathrm{~B}$ & $2,4 \mathrm{~A}$ & $0,1^{\text {ns }}$ & $5,3 \mathrm{~A}$ & $7,6 \mathrm{~A}$ & $69,2 \mathrm{~A}$ & $11,5 \mathrm{~A}$ & $481,0 \mathrm{~A}$ \\
\hline $\mathrm{C}-\mathrm{SOL}$ & $6,3 \mathrm{~A}$ & $2,0 \mathrm{~A}$ & $4,6 \mathrm{~A}$ & $2,2 \mathrm{~A}$ & 0,1 & $5,6 \mathrm{~A}$ & $7,6 \mathrm{~A}$ & $74,0 \mathrm{~A}$ & $7,7 \mathrm{~A}$ & $360,8 \mathrm{~B}$ \\
\hline C-SOM & $6,1 \mathrm{~B}$ & $1,7 \mathrm{~A}$ & $4,3 \mathrm{~B}$ & $2,1 \mathrm{~A}$ & 0,1 & $4,7 \mathrm{~A}$ & $6,4 \mathrm{~B}$ & $74,2 \mathrm{~A}$ & $3,8 \mathrm{~B}$ & $143,3 \mathrm{~B}$ \\
\hline AL-FLE & $5,6 \mathrm{C}$ & $2,1 \mathrm{~A}$ & $2,5 \mathrm{C}$ & $1,5 \mathrm{~B}$ & 0,1 & $2,7 \mathrm{~B}$ & $4,8 \mathrm{C}$ & $57,0 \mathrm{~B}$ & $8,9 \mathrm{~A}$ & $75,1 \mathrm{~B}$ \\
\hline PD & $6,5 \mathrm{~A}$ & $1,0 \mathrm{~B}$ & $2,6 \mathrm{C}$ & $1,4 \mathrm{~B}$ & 0,1 & $3,2 \mathrm{~B}$ & $4,2 \mathrm{C}$ & $76,4 \mathrm{~A}$ & $3,1 \mathrm{~B}$ & $190,1 \mathrm{~B}$ \\
\hline \multirow[t]{2}{*}{$\mathrm{CV}(\%)$} & 3,4 & 19,4 & 10,7 & 16,3 & 20,4 & 17,1 & 12,1 & 8,2 & 27,4 & 23,4 \\
\hline & \multicolumn{10}{|c|}{ Intermediário, camada de $0,05-0,10 \mathrm{~m}$} \\
\hline SAF & $5,9 \mathrm{~A}$ & $2,0 \mathrm{~A}$ & $3,6 \mathrm{~A}$ & $2,2 \mathrm{~A}$ & $0,1^{\text {ns }}$ & $5,2 \mathrm{~A}$ & $7,2 \mathrm{~A}$ & $72,3 \mathrm{~A}$ & $11,4^{\text {ns }}$ & $594,8 \mathrm{~A}$ \\
\hline C-SOL & $6,3 \mathrm{~A}$ & $1,7 \mathrm{~A}$ & $4,1 \mathrm{~A}$ & $2,3 \mathrm{~A}$ & 0,1 & $5,0 \mathrm{~A}$ & $6,7 \mathrm{~A}$ & $74,5 \mathrm{~A}$ & 6,6 & $327,6 \mathrm{~B}$ \\
\hline C-SOM & $6,1 \mathrm{~A}$ & $1,7 \mathrm{~A}$ & $4,1 \mathrm{~A}$ & $2,0 \mathrm{~A}$ & 0,1 & $4,5 \mathrm{~A}$ & $6,2 \mathrm{~A}$ & $73,1 \mathrm{~A}$ & 3,5 & $134,6 \mathrm{~B}$ \\
\hline AL-FLE & $5,4 \mathrm{~B}$ & $1,9 \mathrm{~A}$ & $2,4 \mathrm{~B}$ & $1,6 \mathrm{~B}$ & 0,1 & $2,7 \mathrm{~A}$ & $4,6 \mathrm{~B}$ & $58,2 \mathrm{~B}$ & 9,0 & $73,1 \mathrm{~B}$ \\
\hline PD & $6,4 \mathrm{~A}$ & $1,0 \mathrm{~B}$ & $2,5 \mathrm{~B}$ & $1,4 \mathrm{~B}$ & 0,1 & $3,1 \mathrm{~A}$ & $4,1 \mathrm{~B}$ & $75,2 \mathrm{~A}$ & 3,0 & $184,3 \mathrm{~B}$ \\
\hline \multirow[t]{2}{*}{ CV $(\%)$} & 4,1 & 18,1 & 15,7 & 14,8 & 27,7 & 19,8 & 11,4 & 9,1 & 23,7 & 27,4 \\
\hline & \multicolumn{10}{|c|}{ Fisiogênico, camada de $0,05-0,10 \mathrm{~m}$} \\
\hline SAF & $5,9 \mathrm{~B}$ & $1,8 \mathrm{~A}$ & $3,9 \mathrm{~A}$ & $2,1 \mathrm{~A}$ & $0,1^{\text {ns }}$ & $3,9 \mathrm{~A}$ & $7,4 \mathrm{~A}$ & $72,3 \mathrm{~A}$ & $11,0 \mathrm{~A}$ & $653,3 \mathrm{~A}$ \\
\hline $\mathrm{C}-\mathrm{SOL}$ & $6,3 \mathrm{~A}$ & $1,8 \mathrm{~A}$ & $4,6 \mathrm{~A}$ & $2,2 \mathrm{~A}$ & 0,1 & $3,1 \mathrm{~A}$ & $7,2 \mathrm{~A}$ & $73,7 \mathrm{~A}$ & $6,2 \mathrm{~B}$ & $310,1 \mathrm{~B}$ \\
\hline C-SOM & $5,9 \mathrm{~B}$ & $1,7 \mathrm{~A}$ & $4,3 \mathrm{~A}$ & $2,0 \mathrm{~A}$ & 0,1 & $2,5 \mathrm{~A}$ & $6,7 \mathrm{~A}$ & $70,8 \mathrm{~A}$ & $3,4 \mathrm{~B}$ & $137,5 \mathrm{~B}$ \\
\hline AL-FLE & $5,4 \mathrm{C}$ & $1,5 \mathrm{~A}$ & $2,4 \mathrm{~B}$ & $1,5 \mathrm{~B}$ & 0,1 & $1,7 \mathrm{~A}$ & $4,2 \mathrm{~B}$ & $64,6 \mathrm{~A}$ & $8,8 \mathrm{~A}$ & $73,1 \mathrm{~B}$ \\
\hline PD & $6,5 \mathrm{~A}$ & $0,8 \mathrm{~B}$ & $2,6 \mathrm{~B}$ & $1,2 \mathrm{~B}$ & 0,1 & $1,8 \mathrm{~A}$ & $4,1 \mathrm{~B}$ & $78,1 \mathrm{~A}$ & $2,5 \mathrm{~B}$ & $162,8 \mathrm{~B}$ \\
\hline CV (\%) & 4,5 & 19,6 & 16,8 & 19,3 & 24,3 & 19,5 & 11,7 & 10,3 & 25,6 & 21,7 \\
\hline
\end{tabular}

${ }^{(1)}$ Médias seguidas de letras iguais, nas colunas, não diferem pelo teste Scott-Knott, a 5\% de probabilidade. nsNão significativo a 5\% de probabilidade. SAF, sistema agroflorestal; C-SOL, café cultivado a pleno sol; C-SOM, café sombreado; AL-FLE, cultivo em aleias; PD, sistema plantio direto. 
manutenção do COT no solo. Vários estudos sobre as vias de formação de agregados relatam que os teores de COT foram maiores em agregados biogênicos, principalmente em comparação aos fisiogênicos (Jouquet et al., 2009; Silva Neto et al., 2010; Batista et al., 2013; Loss et al., 2014). Bossuyt et al. (2005) relataram que o conteúdo de COT nos agregados biogênicos foi $22 \%$ maior que o observado em fisiogênicos.

Entre os sistemas avaliados, os maiores teores de COT foram observados nas áreas C-SOL e C-SOM, seguidos pelos observados em SAF e AL-FLE, enquanto os menores teores foram observados na área de $\mathrm{PD}$, em agregados biogênicos. O tempo de implantação dos sistemas pode ajudar a explicar esses resultados, uma vez que as áreas de café são as mais antigas entre as estudadas, com 15 anos de implantação. $\mathrm{Na}$ área sombreada (C-SOM), além da contribuição do café no aporte de $\mathrm{MO}$ ao solo, ainda há a cobertura da gliricídia, e na área a pleno sol (C-SOL), mantém-se uma vegetação de gramíneas nas entrelinhas do café que, além de ajudar na manutenção da umidade do solo, contribui para o aporte de carbono ao solo, tanto em superfície quanto em subsuperfície.

Os teores de carbono orgânico associado a minerais (COam) foram significativamente maiores do que os

Tabela 3. Carbono orgânico total (COT) em agregados biogênicos, intermediários e fisiogênicos, nos diferentes sistemas de manejo agroecológico avaliados ${ }^{(1)}$.

\begin{tabular}{lccl}
\hline Sistema & Biogênico & Intermediário & Fisiogênico \\
& - & 0 & $0,00-0,05 \mathrm{~m}$ \\
& & $20,2 \mathrm{~B}$ & $24,5 \mathrm{~B}$ \\
SAF & $24,6 \mathrm{~B}$ & $27,7 \mathrm{~A}$ & $22,7 \mathrm{~B}$ \\
C-SOL & $30,7 \mathrm{~A}$ & $31,0 \mathrm{~A}$ & $29,7 \mathrm{~A}$ \\
C-SOM & $33,7 \mathrm{~A}$ & $17,2 \mathrm{~B}$ & $17,8 \mathrm{C}$ \\
AL-FLE & $22,0 \mathrm{~B}$ & $14,8 \mathrm{~B}$ & $13,9 \mathrm{C}$ \\
PD & $15,7 \mathrm{C}$ & 14,2 \\
\hline CV $(\%)$ & 15,3 & 14,68 \\
\hline & & $0,05-0,10 \mathrm{~m}$ \\
SAF & $21,4 \mathrm{~B}$ & $23,0 \mathrm{~A}$ & $17,9 \mathrm{~B}$ \\
C-SOL & $29,5 \mathrm{~A}$ & $23,7 \mathrm{~A}$ \\
C-SOM & $21,5 \mathrm{~B}$ & $19,1 \mathrm{~B}$ & $24,8 \mathrm{~A}$ \\
AL-FLE & $15,6 \mathrm{C}$ & $17,0 \mathrm{~B}$ & $14,2 \mathrm{~B}$ \\
PD & $14,7 \mathrm{C}$ & $13,1 \mathrm{~B}$ & $12,0 \mathrm{~B}$ \\
\hline CV $(\%)$ & 16,7 & 13,8 & 24,4 \\
\hline
\end{tabular}

${ }^{(1)}$ Médias seguidas de letras iguais, nas colunas (as comparações nas linhas não foram significativas), não diferem pelo teste Scott-Knott, a 5\% de probabilidade. SAF, sistema agroflorestal; C-SOL, café cultivado a pleno sol; C-SOM, café sombreado; AL-FLE, cultivo em aleias; PD, sistema de plantio direto. de carbono orgânico particulado (COp), em todos os sistemas de manejo, independentemente do tipo de agregado e profundidade (Tabela 4). Os maiores teores de COam podem ser explicados pela maior proteção da matéria orgânica proporcionada pelos minerais de argila e silte, que os tornam menos suscetíveis ao tipo de manejo. Estes resultados corroboram estudos com agregados provenientes de diferentes vias de formação (Silva Neto et al., 2010; Batista et al., 2013; Loss et al., 2014).

$\mathrm{Na}$ comparação entre os sistemas, assim como o observado quanto ao COT, os maiores teores de COp foram obtidos nas áreas de C-SOL e C-SOM, em agregados biogênicos e, na área de C-SOL, até $0,05 \mathrm{~m}$ de profundidade, em agregados fisiogênicos. O COp representa a fração mais lábil da matéria orgânica, que é mais sensível ao revolvimento do solo e facilmente mineralizada caso o sistema de manejo

Tabela 4. Carbono orgânico particulado (COp) e carbono orgânico associado a minerais (COam) em agregados biogênicos, intermediários e fisiogênicos, nos diferentes sistemas de manejo agroecológico avaliados ${ }^{(1)}$.

\begin{tabular}{|c|c|c|c|c|}
\hline \multirow[t]{3}{*}{ Sistema } & \multicolumn{2}{|c|}{ Camada $0,00-0,05 \mathrm{~m}$} & \multicolumn{2}{|c|}{ Camada $0,05-0,10 \mathrm{~m}$} \\
\hline & $\mathrm{COp}$ & COam & $\mathrm{COp}$ & COam \\
\hline & \multicolumn{4}{|c|}{ - } \\
\hline & \multicolumn{4}{|c|}{ Biogênicos } \\
\hline SAF & $1,78 \mathrm{Bb}$ & $22,91 \mathrm{Aa}$ & $1,62 \mathrm{Bb}$ & $19,82 \mathrm{Ba}$ \\
\hline C-SOL & $2,87 \mathrm{Ab}$ & $27,03 \mathrm{Aa}$ & $2,97 \mathrm{Ab}$ & $26,56 \mathrm{Aa}$ \\
\hline C-SOM & $2,15 \mathrm{Ab}$ & $31,14 \mathrm{Aa}$ & $1,65 \mathrm{Bb}$ & $19,88 \mathrm{Ba}$ \\
\hline AL-FLE & $2,18 \mathrm{Ab}$ & $17,08 \mathrm{Ba}$ & $1,11 \mathrm{Cb}$ & $14,54 \mathrm{Ca}$ \\
\hline PD & $1,08 \mathrm{Bb}$ & $14,75 \mathrm{Ba}$ & $0,81 \mathrm{Cb}$ & $13,9 \mathrm{Ca}$ \\
\hline \multirow[t]{2}{*}{ CV (\%) } & 24,46 & 18,4 & 17,8 & 18,08 \\
\hline & \multicolumn{4}{|c|}{ Intermediários } \\
\hline SAF & $2,07 \mathrm{Ab}$ & $17,47 \mathrm{Ba}$ & $1,09 \mathrm{Bb}$ & $21,94 \mathrm{Aa}$ \\
\hline C-SOL & $1,95 \mathrm{Ab}$ & $25,88 \mathrm{Aa}$ & $2,36 \mathrm{Ab}$ & $21,39 \mathrm{Aa}$ \\
\hline C-SOM & $1,56 \mathrm{Ab}$ & $29,52 \mathrm{Aa}$ & $1,12 \mathrm{Bb}$ & $17,98 \mathrm{Aa}$ \\
\hline AL-FLE & $1,68 \mathrm{Ab}$ & $15,58 \mathrm{Ba}$ & $1,27 \mathrm{Bb}$ & $15,8 \mathrm{Ba}$ \\
\hline PD & $0,83 \mathrm{Bb}$ & $14,26 \mathrm{Ba}$ & $0,81 \mathrm{Bb}$ & $12,35 \mathrm{Ba}$ \\
\hline \multirow[t]{2}{*}{ CV (\%) } & 23,4 & 21,5 & 15,27 & 16,07 \\
\hline & \multicolumn{4}{|c|}{ Fisiogênicos } \\
\hline SAF & $0,99 \mathrm{Bb}$ & $19,85 \mathrm{Ba}$ & $0,97 \mathrm{Ab}$ & $16,95 \mathrm{Ba}$ \\
\hline C-SOL & $2,36 \mathrm{Ab}$ & $20,35 \mathrm{Ba}$ & $1,63 \mathrm{Ab}$ & $23,25 \mathrm{Aa}$ \\
\hline C-SOM & $1,48 \mathrm{Bb}$ & $28,22 \mathrm{Aa}$ & $1,24 \mathrm{Ab}$ & $15,49 \mathrm{Ba}$ \\
\hline AL-FLE & $1,15 \mathrm{Bb}$ & $15,74 \mathrm{Ca}$ & $0,97 \mathrm{Ab}$ & $13,33 \mathrm{Ba}$ \\
\hline PD & $0,43 \mathrm{Bb}$ & $12,7 \mathrm{Ca}$ & $0,86 \mathrm{Ab}$ & $11,22 \mathrm{Ba}$ \\
\hline $\mathrm{CV}(\%)$ & 16,6 & 16,4 & 23,2 & 25,4 \\
\hline
\end{tabular}


não seja conservacionista, o que está em conformidade com os maiores teores dessa fração, observados nas áreas com maior tempo de manejo agroecológico, no presente trabalho. De acordo com Bayer et al. (2004), o acúmulo de COp representa um benefício ambiental dependente da continuidade de sistemas de preparo mínimo do solo, como o PD.

Em área de pastagem com 30 anos e em área de floresta, Loss et al. (2014) verificaram maiores conteúdos de COp em agregados biogênicos e fisiogênicos do que os observados sob cultivo (PD e PC), o que mostra a importância do elevado aporte de material orgânico ao solo, seja via serapilheira ou sistema radicular, para esta fração.

Batista et al. (2013) relataram que os teores de COp, em sistema de integração lavoura-pecuária, foram mais sensíveis ao manejo do que os de COT, e que a fração particulada da matéria orgânica é potencial indicadora da qualidade da MOS em razão de alterações provenientes do manejo.

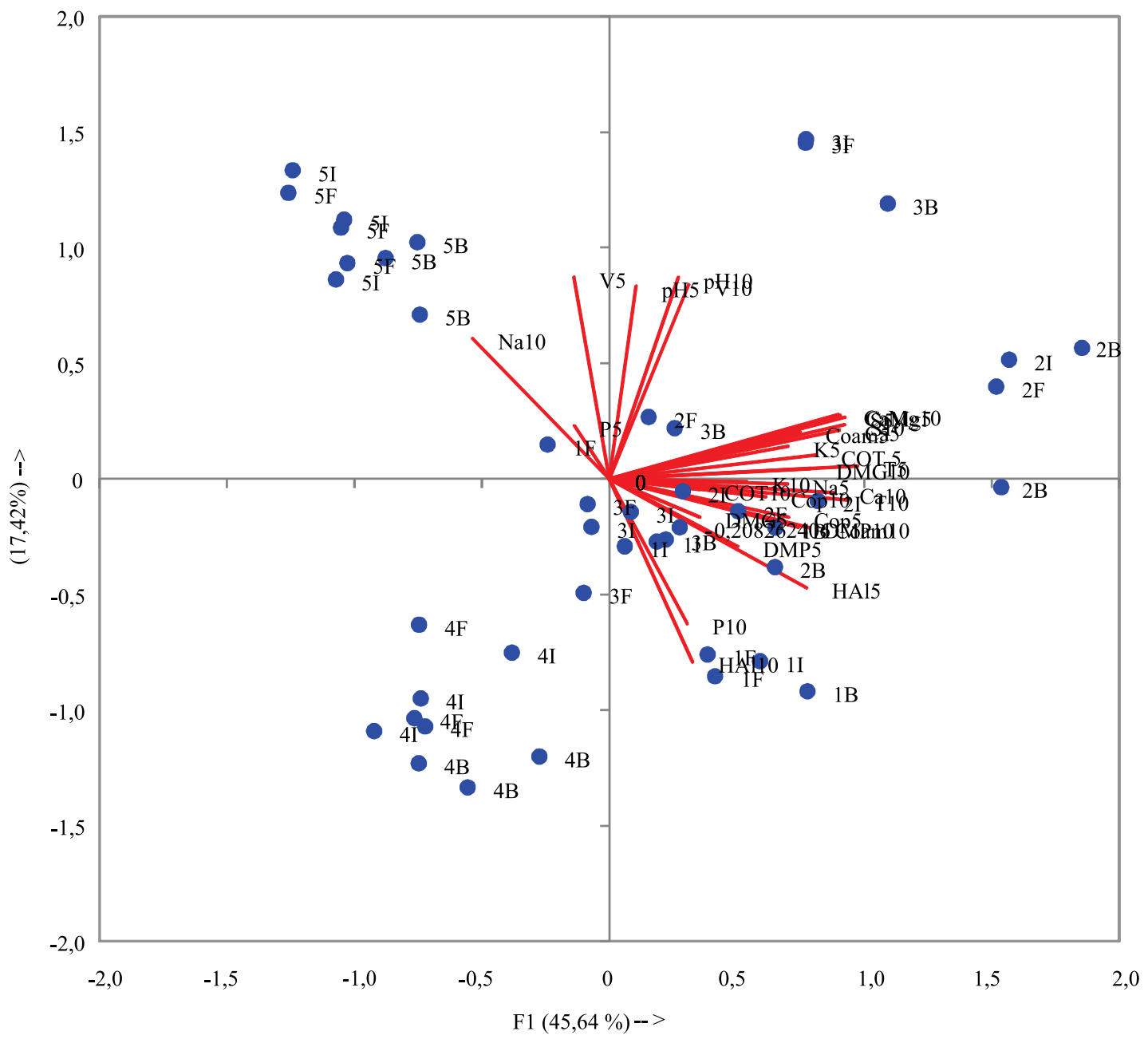

Figura 2. Gráfico biplot da análise de componentes principais das características químicas do solo e físicas de agregados biogênicos, intermediários e fisiogênicos, nos diferentes sistemas de manejo agroecológico avaliados, às camadas de 0,00-0,05 e 0,05-0,10 m: B, agregados biogênicos; I, agregados intermediários; F, agregados fisiogênicos; 1, SAF, sistema agroflorestal; 2, C-SOL, café cultivado a pleno sol; 3, C-SOM, café sombreado; 4, AL-FLE, cultivo em aleias; 5, PD, sistema plantio direto; DMP, diâmetro médio ponderado; DMG, diâmetro médio geométrico; COT, carbono orgânico total; COp, carbono orgânico particulado; COam, carbono orgânico mineral; $\mathrm{CaMg}$, cálcio+magnésio; $\mathrm{Ca}$, cálcio; $\mathrm{Na}$, sódio trocável; $\mathrm{K}$, potássio trocável; P, fósforo disponível; S, soma de bases; T, valor T; V, saturação por bases (\%); 5, camada de 0,00-0,05 m; e 10 , camada de $0,05-0,10 \mathrm{~m}$. 
A análise de componentes principais foi realizada a partir das interpretações feitas na distribuição dos atributos químicos e físicos do solo, nos diferentes tipos de agregados (Figura 2). O gráfico ACP mostra grande separação em três grupos, que têm 63,03\% da variância total explicada por dois componentes (F1, 45,64\%, e F2, 17,42\%). Houve clara separação das áreas PD (5) e AL-FLE (4) das demais áreas estudadas. Nessas duas áreas, somente o conteúdo de $\mathrm{Na}$, a $0,05-0,10 \mathrm{~m}$ de profundidade, apresentou correlação com o tipo de manejo. As áreas com maior tempo de implementação do sistema conservacionista e menor uso de práticas culturais - SAF (1), C-SOL (2) e C-SOM (3) - foram similares e se correlacionaram à maioria dos atributos químicos e físicos avaliados. Esta similaridade esteve associada aos elevados teores de COT e DMP verificados nesses sistemas.

\section{Conclusões}

1. Nos sistemas conservacionistas avaliados, o tipo de manejo não interfere significativamente nas proporções relativas dos diferentes tipos de agregados, e os intermediários apresentam as maiores contribuições para a massa total de agregados.

2. Sistemas conservacionistas com maior tempo de implantação e menor emprego de práticas culturais favorecem o acúmulo e a manutenção do carbono no solo, com consequentes aumentos dos valores de diâmetro médio ponderado e de diâmetro médio geométrico dos agregados, bem como da disponibilidade de $\mathrm{Ca}$ e $\mathrm{Mg}$.

3. Os sistemas conservacionistas avaliados favorecem o acúmulo de carbono orgânico associado a minerais, em relação ao de carbono orgânico particulado.

\section{Agradecimentos}

À Coordenação de Aperfeiçoamento de Pessoal de Nível Superior (Capes) e à Fundação de Amparo à Pesquisa do Estado do Rio de Janeiro (Faperj), pelo apoio financeiro.

\section{Referências}

ALMEIDA, D.L. de; RIBEIRO, R. de L.D.; GUERRA, J.G.M. Sistema integrado de produção agroecológica ("Fazendinha Agroecológica Km 47"). In: SIMPÓSIO DE AGRICULTURA ECOLÓGICA, 2.; ENCONTRO DE AGRICULTURA
ORGÂNICA, 1., 1999, Guaíba, RS. Anais. Guaíba: Agropecuária, 1999. p.152-159.

ARGENTON, J.; ALBUQUERQUE, J.A.; BAYER, C.; WILDNER, L. do P. Comportamento de atributos relacionados com a forma da estrutura de Latossolo Vermelho sob sistemas de preparo e plantas de cobertura. Revista Brasileira de Ciência do Solo, v.29, p.425435, 2005. DOI: 10.1590/S0100-06832005000300013.

BATISTA, I. Atributos edáficos e fauna do solo em áreas de integração lavoura-pecuária no bioma Cerrado, Mato Grosso do Sul. 2011. 86p. Dissertação (Mestrado) - Universidade Federal Rural do Rio de Janeiro, Seropédica.

BATISTA, I.; CORREIA, M.E.F.; PEREIRA, M.G.; BIELUCZYK, W.; SCHIAVO, J.A.; MELLO, N.A. de. Caracterização dos agregados em solos sob cultivo no Cerrado, MS. Semina: Ciências Agrárias, v.34, p.1535-1548, 2013.

BAYER, C.; MARTIN-NETO, L.; MIELNICZUK, J.; PAVINATO, A. Armazenamento de carbono em frações lábeis da matéria orgânica de um Latossolo Vermelho sob plantio direto. Pesquisa Agropecuária Brasileira, v.39, p.677-683, 2004. DOI: 10.1590/ S0100-204X2004000700009.

BOSSUYT, H.; SIX, J.; HENDRIX, P.F. Protection of soil carbon by microaggregates within earthworm casts. Soil Biology \& Biochemistry, v.37, p.251-258, 2005. DOI: 10.1016/j. soilbio.2004.07.035.

BRADY, N.C.; WEIL, R.R. The nature and properties of soils. $3^{\text {rd }}$ ed. New York: Bookman, 2013. 922p.

BRONICK, C.J.; LAL, R. Soil structure and management: a review. Geoderma, v.124, p.3-22, 2005. DOI: 10.1016/j. geoderma.2004.03.005.

BRUSSAARD, L.; PULLEMAN, M.M.; OUÉDRAOGO, É.; MANDO, A.; SIX, J. Soil fauna and soil function in the fabric of the food web. Pedobiologia, v.50, p.447-462, 2007. DOI: 10.1016/j.pedobi.2006.10.007.

BULLOCK, P.; FEDEROFF, N.; JONGERIUS, A.; STOOPS, G.; TURSINA, T. Handbook for soil thin section description. Albrighton: Waine Research, 1985. 152p.

CAMBARDELLA, C.A.; ELLIOTT, E.T. Particulate soil organicmatter changes across a grassland cultivation sequence. Soil Science Society of American Journal, v.56, p.777-783, 1992. DOI: $10.2136 /$ sssaj 1992.03615995005600030017x.

CLAESSEN, M.E.C. (Org.). Manual de métodos de análise de solo. 2.ed. rev. e atual. Rio de Janeiro: Embrapa-CNPS, 1997. 212p. (Embrapa-CNPS. Documentos, 1).

FERNÁNDEZ,R.; QUIROGA,A.;ZORATI,C.; NOELLEMEYER, E. Carbon contents and respiration rates of aggregate size fractions under no-till and conventional tillage. Soil and Tillage Research, v.109, p.103-109, 2010. DOI: 10.1016/j.still.2010.05.002.

JOUQUET, P.; ZANGERLE, A.; RUMPEL, C.; BRUNET, D.; BOTTINELLI, N.; DUC, T.T. Relevance and limitations of biogenic and physicogenic classification: a comparison of approaches for differentiating the origin of soil aggregates. European Journal of Soil Science, v.60, p.1117-1125, 2009. DOI: 10.1111/j.13652389.2009.01168.x. 
LIMA, H.V. de; OLIVEIRA, T.S. de; OLIVEIRA, M.M. de; MENDONÇA, E.S.; LIMA, P.J.B.F. Indicadores de qualidade do solo em sistemas de cultivo orgânico e convencional no semi-árido cearense. Revista Brasileira de Ciência do Solo, v.31, p.10851098, 2007. DOI: 10.1590/S0100-06832007000500024.

LOSS, A.; PEREIRA, M.G.; COSTA, E.M.; BEUTLER, S.J. Soil fertility, physical and chemical organic matter fractions, natural ${ }^{13} \mathrm{C}$ and ${ }^{15} \mathrm{~N}$ abundance in biogenic and physicogenic aggregates in areas under different land use systems. Soil Research, v.52, p.685697, 2014. DOI: $10.1071 /$ SR14045.

MATIELlO, J.B.; GARCIA, A.W.R.; ALMEIDA, S.R. Adubos, corretivos e defensivos para a lavoura cafeeira: indicações de uso. Brasília: Ministério da Agricultura, Pecuária e Abastecimento, 2006. 89p.

MULUMBA, L.N.; LAL, R. Mulching effects on selected soil properties. Soil and Tillage Research, v.98, p.106-111, 2008. DOI: 10.1016/j.still.2007.10.011.

NEVES, M.C.P.; GUERRA, J.G.M.; CARVALHO, S.R.; RIBEIRO, R.L.D.; ALMEIDA, D.L. Sistema integrado de produção agroecológica ou Fazendinha Agroecológica do Km 47. In: AQUINO, A.; ASSIS, R.L. de (Ed.). Agroecologia: princípios e técnicas para uma agricultura orgânica sustentável. Brasília: Embrapa Informação tecnológica; Seropédica, RJ: Embrapa Agrobiologia, 2005. p.147-172.

NOELLEMEYER, E.; FRANK, F.; ALVAREZ, C.; MORAZZO, G.; QUIROGA, A. Carbon contents and aggregation related to soil physical and biological properties under a land-use sequence in the semiarid region of central Argentina. Soil and Tillage Research, v.99, p.179-190, 2008. DOI: 10.1016/j.still.2008.02.003.

PULLEMAN, M.M.; MARINISSEN, J.C.Y. Physical protection of mineralizable $\mathrm{C}$ in aggregates from long-term pasture and arable soil. Geoderma, v.120, p.273-282, 2004. DOI: 10.1016/j. geoderma.2003.09.009.

PULLEMAN, M.M.; SIX, J.; VAN BREEMEN, N.; JONGMANS, A.G. Soil organic matter distribution and microaggregate characteristics as affected by agricultural management and earthworm activity. European Journal of Soil Science, v.56, p.453-467, 2005. DOI: 10.1111/j.13652389.2004.00696.x.

RILLIG, M.C.; MUMMEY, D.L. Mycorrhizas and soil structure. New Phytologist, v.171, p.41-53, 2006. DOI: 10.1111/j.14698137.2006.01750.x.

SANTOS, H.G. dos; JACOMINE, P.K.T.; ANJOS, L.H.C. dos; OLIVEIRA, V.A. de; LUMBRERAS, J.F.; COELHO, M.R.; ALMEIDA, J.A. de; CUNHA, T.J.F.; OLIVEIRA, J.B. de. Sistema brasileiro de classificação de solos. 3.ed. rev. e ampl. Brasília: Embrapa, 2013. 353p.

SCHRADER, S.; ZHANG, H. Earthworm casting: stabilization or destabilization of soil structure? Soil Biology and Biochemistry, v.29, p.469-475, 1997. DOI: 10.1016/S0038-0717(96)00103-4.

SILVA NETO, E.C.; PEREIRA, M.G.; FERNANDES, J.C.F. Gênese e estabilidade de agregados sob diferentes coberturas vegetais, Pinheiral - RJ. In: SIMPÓSIO DE PESQUISA EM MATA ATLÂNTICA, 2., 2012, Engenheiro Paulo de Frontin. Anais. Engenheiro Paulo de Frontin: Instituto Zoobotânico de Morro Azul, 2012. p.72-74.

SILVA NETO, L. de F. da; SILVA, I. de F. da; INDA, A.V.; NASCIMENTO, P.C. do; BORTOLON, L. Atributos físicos e químicos de agregados pedogênicos e de coprólitos de minhocas em diferentes classes de solos da Paraíba. Ciência e Agrotecnologia, v.34, p.1365-1371, 2010.

SIX, J.; BOSSUYT, H.; DEGRYZE, S.; DENEF, K. A history of research on the link between (micro)aggregates, soil biota, and soil organic matter dynamics. Soil and Tillage Research, v.79, p.7-31, 2004. DOI: 10.1016/j.still.2004.03.008.

YEOMANS, J.C.; BREMNER, J.M. A rapid and precise method for routine determination of organic carbon in soil. Communications in Soil Science and Plant Analysis, v.19, p.1467-1476, 1988. DOI: $10.1080 / 00103628809368027$. 